\title{
Oscillating Hypothyroidism and Hyperthyroidism: A Rare Autoimmune Syndrome
}

\author{
Gabriel Melki ${ }^{\mathrm{a}}$,, FNU Komal ${ }^{\mathrm{a}}$, Linda Laham ${ }^{\mathrm{a}}$, Gres Karimª, Vinod Kumara, \\ Sushant Nanavatia ${ }^{\text {a }}$ Mourad Ismail ${ }^{\mathrm{a}}$
}

\begin{abstract}
Graves' disease is an autoimmune thyroid disorder with overproduction of thyroid hormones secondary to the production of thyroid-stimulating hormone receptor (TSH-R) stimulating antibodies (TSAbs). It is very common for Graves' disease patients to convert to a hypothyroid state especially in end-stage Graves' disease or after iodine ablation. These patients require lifelong thyroxin replacement therapy. In this case report, we describe a rare phenomenon known as "oscillating hypo- and hyperthyroidism". In this rare entity of autoimmune endocrine disorder patients with hypothyroidism spontaneously convert to Graves' disease.
\end{abstract}

Keywords: Graves' disease; Autoimmune; Oscillating hypothyroidism and hyperthyroidism; Oscillating hypo- and hyperthyroidism

\section{Introduction}

Autoimmune thyroid diseases tend to move thyroid hormones in one direction. Graves' disease is a well known cause for hyperthyroidism. While transforming from Graves' hyperthyroidism into hypothyroidism is not uncommon, having both conditions simultaneously forms a unique combination and a challenge to medical management. We present a case of a 37-year-old woman who had oscillating hypothyroidism and hyperthyroidism, and a surgical ablation of the gland was recommended as definitive treatment.

\section{Case Report}

A 37-year-old woman with the PMH of type 1 diabetes, Hashimoto thyroiditis being treated with levothyroxine and autoim-

Manuscript submitted May 28, 2019, accepted June 8, 2019

aDepartment of Internal Medicine, St. Joseph's University Medical Center, Paterson, NJ 07503, USA

${ }^{b}$ Corresponding Author: Gabriel Melki, Department of Internal Medicine, St. Joseph's University Medical Center, Paterson, NJ 07503, USA.

Email: gabriel.melki@mail.mcgill.ca

doi: https://doi.org/10.14740/jem583 mune pancreatitis came to the emergency department (ED) with complaints of palpitations, tremors, and anxiety. The patient's vitals were: heart rate (HR): 110, temperature: $100 \mathrm{~F}$, respiratory rate (RR): 18/min, blood pressure (BP): 160/90. Physical exam was positive for exophthalmos and pretibial myxedema. Patient labs revealed: high free $\mathrm{T} 3 \mathrm{of} 4.83 \mathrm{pg} / \mathrm{mL}$ and high free $\mathrm{T} 4 \mathrm{of} 2.18 \mathrm{ng} / \mathrm{dL}$ and undetectable thyroid-stimulating hormone (TSH) of $0.015 \mathrm{uIU} / \mathrm{mL}$ (Supplementary 1). Thyroid ultrasound revealed diffuse enlargement of the thyroid gland and thyroid isotope scan revealed diffusely increased uptake, suggestive of Graves' disease and therefore TSH receptor (TSH-R) stimulating antibodies (TSAbs) were ordered which were positive.

Prior records of this patient revealed that the patient's TSH was high $(57.143 \mathrm{uIU} / \mathrm{mL})$, free T3 $(2.12 \mathrm{pg} / \mathrm{mL})$ and free T4 $(0.42 \mathrm{ng} / \mathrm{dL})$ were low and patient was being treated with levothyroxine, but based on new signs and symptoms and new investigation the patient was diagnosed with Graves' disease. Patient was offered various treatment options like thyroid ablation and surgery; however she refused any kind of intervention and was treated with methimazole.

After a few months, the patient developed signs and symptoms of hypothyroidism. Labs were drawn and revealed that the TSH was elevated at $62.993 \mathrm{uIU} / \mathrm{mL}$, free T3 and free T4 were low at $2.01 \mathrm{pg} / \mathrm{mL}$ and $0.7 \mathrm{ng} / \mathrm{dL}$ respectively and TSH$\mathrm{R}$ blocking antibodies (TBAbs) were sent and were positive. These symptoms and laboratory data were suspicious of hypothyroidism. The patient's change from hyper- to hypothyroidism prompted us to diagnose the patient with oscillating hypothyroidism and hyperthyroidism.

\section{Discussion}

Oscillating hypothyroidism and hyperthyroidism is an autoimmune entity due to the production of two types of thyrotropin receptor (TSH-R) autoantibodies found in immune disorders of the thyroid: TSAb and TBAb. TSAb is responsible for overactive thyroid gland causing Graves's disease, while TBAb causes hypothyroidism by blocking endogenous TSH.

In one study by Takeda et al [1], they described that both types of TSH-R antibodies can coexist in one patient and that the thyroid function and clinical picture may change according to the balance of the type antibodies seen in the patient's system.

In another study by McLachlan et al [2], they concluded that the balance between TBAb and TSAb can be shifted be- 
cause of treatment with thyroid hormone replacement therapy or antithyroid treatment. Both therapies can alter the level of thyroid antibodies and can easily swing balance from hypo- to hyperthyroidism and vice versa.

This disease is difficult to treat as treatment of hypothyroidism can lead to overactive hyperthyroid. A block and replace regimen may improve stability over the short to intermediate term, but this is not a definitive therapy and requires long-term close monitoring. Definitive therapy includes both thyroidectomy and radioactive iodine, which has the disadvantage in that it can only be performed in the hyperthyroid phase [3].

The patient was educated about all available treatment options; however, the patient refused thyroidectomy and opted for treatment with antithyroid medications. The patient was specifically treated with methimazole. Without definitive treatment such as surgery or thyroid ablation, management can be challenging and close monitoring of the patient along with regular thyroid function test will be required for ongoing follow-up [3].

\section{Conclusions}

Physicians encounter cases of both hypothyroidism and hyperthyroidism throughout their careers; however they should always remain attentive to both the patient's symptoms and laboratory values as cases of oscillating hypo- and hyperthyroidism are rare but need strict rigorous monitoring. Physicians must be careful in treating these cases, as patients can easily switch between hypo- and hyperthyroid states. The learning points include: 1) Patients with these autoimmune phenomena should be monitored with TSAb and TBAb along with thyroid function tests; and 2) Definitive therapy for oscillating hypothyroid and hyperthyroid is thyroidectomy (during any state of disease) or radioactive iodine ablation, while the patient is in the hyperthyroid state.

\section{Acknowledgments}

We thank Dr. Patrick Michael, Program Director of the Internal
Medicine Residency for his continuous support and guidance.

\section{Financial Disclosure}

No funding was provided to any of the authors.

\section{Conflict of Interest}

The authors declare that they have no conflict of interest.

\section{Informed Consent}

Informed consent was obtained from the patient for this case report.

\section{Author Contributions}

All authors contributed to the revision and approval of the manuscript.

\section{References}

1. Takeda K, Takamatsu J, Kasagi K, Sakane S, Ikegami Y, Isotani H, Majima T, et al. Development of hyperthyroidism following primary hypothyroidism: a case report with changes in thyroid-related antibodies. Clin Endocrinol (Oxf). 1988;28(4):341-344.

2. McLachlan SM, Rapoport B. Thyrotropin-blocking autoantibodies and thyroid-stimulating autoantibodies: potential mechanisms involved in the pendulum swinging from hypothyroidism to hyperthyroidism or vice versa. Thyroid. 2013;23(1):14-24.

3. Wong M, Inder WJ. Alternating hyperthyroidism and hypothyroidism in Graves' disease. Clin Case Rep. 2018;6(9):1684-1688. 